\title{
A robust tracking system for COVID-19 like pandemic using advanced hybrid technologies
}

\author{
Shibli Nisar ${ }^{1} \cdot$ Muhammad Ali Zuhaib $^{1} \cdot$ Abasin Ulasyar $^{1} \cdot$ Muhammad Tariq $^{2}$
}

Received: 21 December 2020 / Accepted: 30 March 2021 / Published online: 28 May 2021

(c) The Author(s), under exclusive licence to Springer-Verlag GmbH Austria, part of Springer Nature 2021

\begin{abstract}
In order to track patients in coronavirus (COVID-19) like pandemic, this paper proposes a novel model based on hybrid advance technologies, which is capable to trace and track COVID-19 affectees with high accuracy. The hybrid technologies include, cellular, cyber and low range wireless technologies. This technique is capable to trace patients through call data record using cellular technology, voice over Internet protocol calls using cyber technology and physical contact without having a call history using low range wireless technologies. The proposed model is also capable to trace COVID-19 suspects. In addition to tracking, the proposed model is capable to provide surveillance capability as well by geo tagging the patients. In case of any violation by the patients an alert is sent to the concerned department. The proposed model is cost effective and privacy preserved as the entire process is carried out under the umbrella of a concerned government department. The potential outcomes of the proposed model are tracking of COVID-19 patients, monitoring of isolated patients, tracking of suspected ones and inform the mass about the safest path to use.
\end{abstract}

Keywords CDR · Coronavirus · Control · COVID-19 · Cellular forensics · Hidden pattern $\cdot$ Privacy preservation

\section{Introduction}

Coronavirus (COVID-19) is a contagious and transmissible disease that is caused by coronavirus [1]. The virus has spread rapidly throughout the world and infected millions of people worldwide [2-4]. Different misconceptions associated with the virus has made the COVID-19 more frightening [5]. COVID-19 is basically a member of ribonucleic acid (RNA) based virus family, which is called as coronaviridae [6]. Until

\footnotetext{
Shibli Nisar

shiblinisar@mcs.edu.pk

1 National University of Sciences and Technology (NUST), Islamabad 44000, Pakistan

2 National University of Computer and Emerging Sciences, Islamabad, Pakistan
} 
there is availability of a safe and secure vaccine available for treatment of COVID19 patients, the curve of the outbreak needs to be flattened. For this purpose, many governments have implemented strict lockdowns in their respective countries, which result into complete closure of their borders for any type of travelling by the people. Operational and financial activities of various socioeconomic sectors are badly disturbed. These include pharmaceutical and healthcare industries, education, tourism sector, oil and petroleum industry, agriculture, media and entertainment industry, finance industry, aviation, housing and real estate sector, manufacturing sector and sports industry [7-18]. Moreover due to lockdown measures, the normal routine life is severely affected [3].

On the other hand, researchers around the world are rigorously involved in the development of an effective drug or vaccine to fight the COVID-19 outbreak. Although different vaccines and drugs are still passing through various testing stages before they can be available worldwide, social distancing, quarantining and tracking based strategies are useful to control and reduce the effects of COVID-19 [19-24]. This paper proposes a novel model to control the spread of coronavirus using hybrid advance technologies. These hybrid technologies include call data record (CDR) using cellular technology, physical contact without having a call history using low range wireless technologies such as Bluetooth, and voice over Internet protocol (VOIP) calls using cyber technologies. A CDR is basically a log file that is maintained by different telecom operators. It contains different types of data, which include latitude and longitude details of base transceiver station (BTS), type of call, international mobile equipment identity (IMEI), detail of cell site, numbers of source and destination, and calls duration made and received at a certain time $[13,25,26]$. Since CDR analysis is a very useful tool to trace and investigate criminals, therefore, it is widely used throughout the world by different law enforcement agencies (LEA) [27-29]. This technique is widely used due to its promising results $[27,30,31]$.

COVID-19 patients are usually tracked by using contact tracing. The contact tracing strategy utilizes an exposure notifications system (ENS). ENS is a framework which is developed by Google and Apple to trace infected people during COVID-19 outbreak [32-36]. Various traditional contact tracing techniques are coupled with ENS by the government of a particular country. This fusion can be realized with the help of logging the encounters among ENS users by using their Android or iOS smartphones. ENS is a decentralized reporting based protocol, which is built on a combination of Bluetooth low energy (BLE) technology and privacy-preserving cryptography.

Nowadays, trend of VOIP calls are exponentially increasing due to availability, reliability and speed of the Internet. Since calls made on VOIP are not registered in CDR $\log$, therefore, it is not possible to track such cases using call data record analysis (CDRA). In addition to this, if call is made on such apps and mobile is not taken along then the contact tracing strategy fails to track the COVID-19 patients. In order to overcome these limitations, the proposed model includes additional tracking feature to track COVID-19 patients based on the VOIP calls data log. The proposed model is capable of monitoring and tracing patients with and without call history of COVID-19 patients along with the calls made on the VOIP as shown in the Fig. 1.

Rest of the paper is organized as follow: Sect. 2 presents the existing work and limitations. Proposed tracing model is covered in Sect. 3. Section 4 presents the con- 


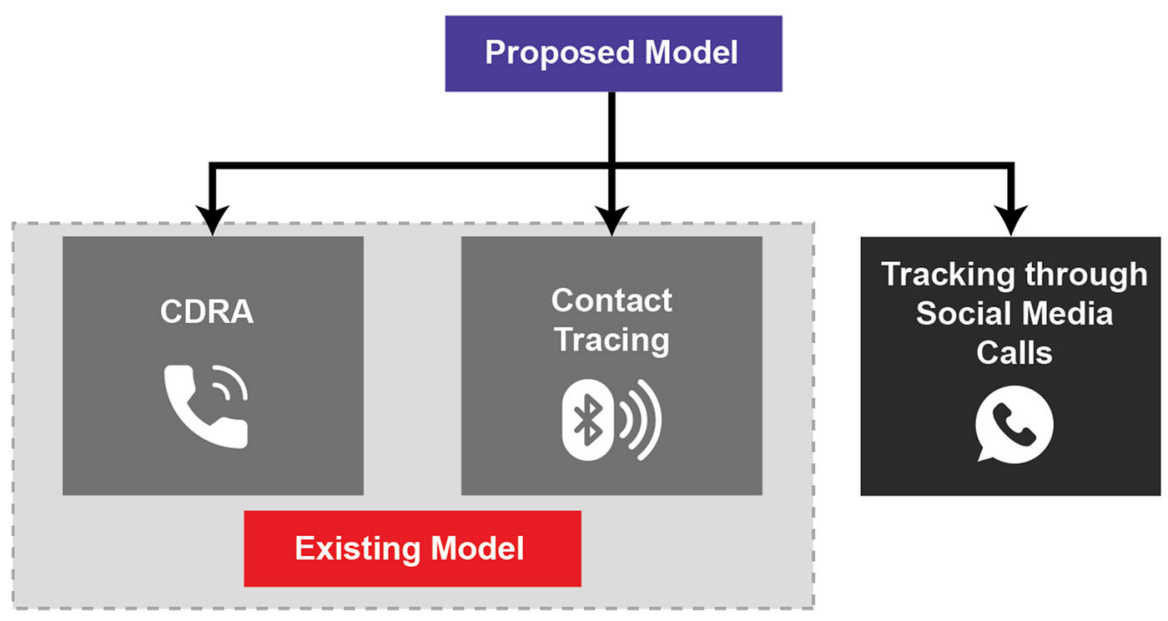

Fig. 1 Block diagram of the proposed model

clusive remarks along with the potential outcomes of the proposed model, and some future directions.

\section{Related work}

This section presents a brief description of the existing works done on tracking system of COVID-19 patients along with their limitations. These limitations are overcome by the proposed model.

\subsection{CDRA framework}

A model that can immediately locate the patients by using the geo position is needed to reduce the spread of COVID-19 cases. This can be achieved with the help of call history of a patient. The spread can be easily minimized within few weeks by tracking, isolation and monitoring of COVID-19 patients. Ignoring the instructions at the start will damage a lot. CDRA can be easily implemented to avoid the spread. In order to reach the suspects who met the COVID-19 patients can be easily tracked through their call history using CDRA. COVID-19 outbreak can be easily minimized or stop using this model. In addition to tracking, the proposed model is capable to provide surveillance capability as well by geo tagging the patients. In case of any violation by the patients an alert is sent to the concerned department. By doing so, one can easily avoid the coronavirus spread by isolating themselves. Working of the CDRA based tracking model is shown in Fig. 2.

CDR can also incorporate the messaging metadata of short messaging services (SMS) and any other transmission of official communication. Nevertheless, the privacy remains intact by not revealing the contents of the messages and calls through the CDR. The data of calls and messages at different instances shows their occurrence. It helps 


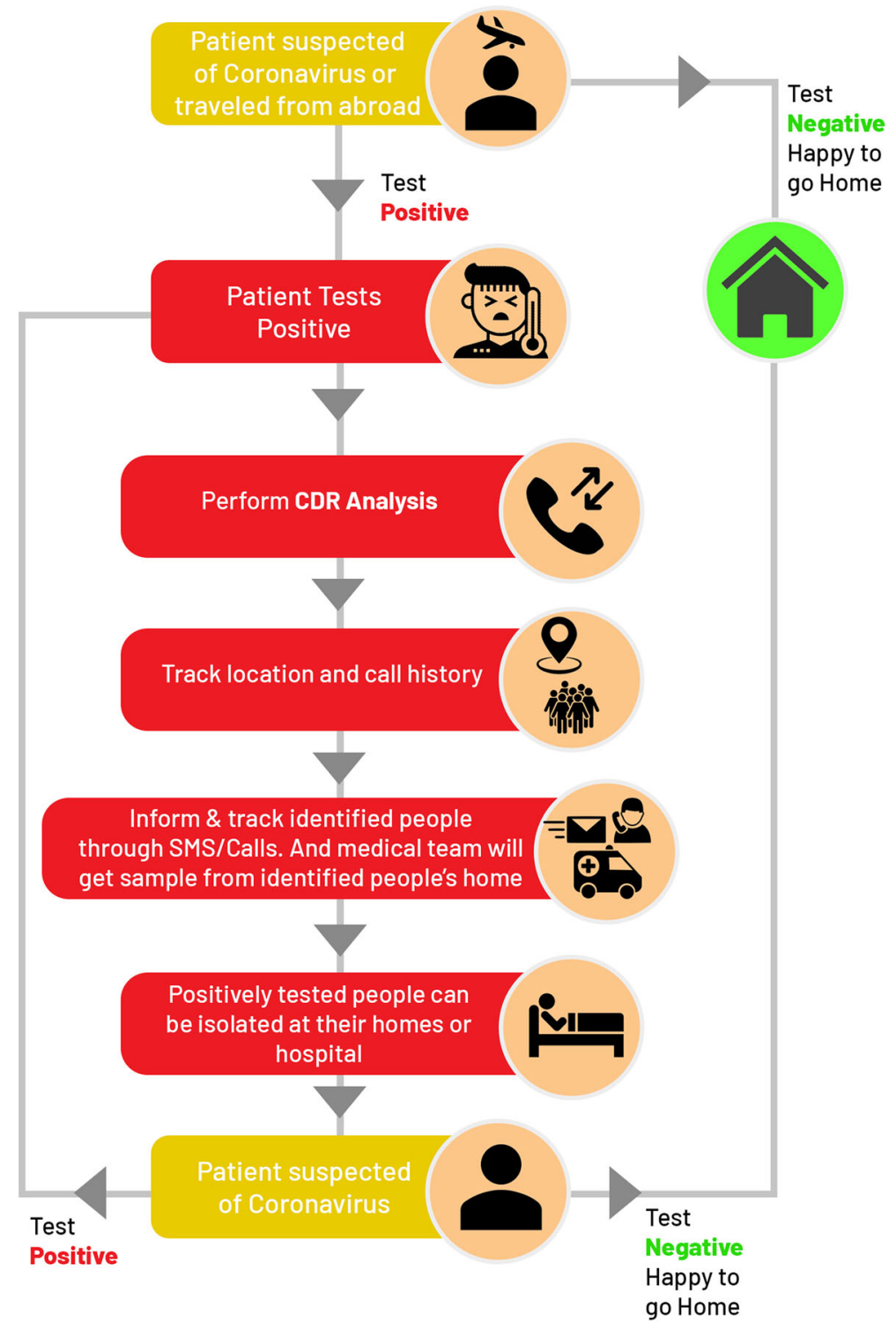

Fig. 2 Detail flowchart of CDRA model

to measure basic properties of calls and messages. At first, CDRA has to be performed for all the COVID-19 infected people in a particular country. Furthermore, it has to be extended for those infected people who travelled from abroad to that specific country. The concerned authorities will be notified by hospital management in case a person is declared a COVID-19 patient. Consequently, a CDRA will be triggered on the active SIM of the patient's smartphone with the permission of the affected patient. A 
concerned team of a government authority will contact and trace for suspected people with whom the COVID-19 patient might have met and interacted in one way or other. The ambulances will be dispatched to the homes of suspected patients to collect the samples for their COVID-19 tests. The suspected people will be advised to completely quarantine at their homes till the results of COVID-19 tests are declared to them. If a particular person is diagnosed as COVID-19 patient, then the patient will be sent to the hospital. In this way, same procedure will be followed for all those individuals with whom the affected patient has come in contact. For the overall operation of this model, a case study has already been performed in [37].

\subsubsection{Limitations of CDRA}

This section discusses the limitation of CDRA. These limitations have restricted the utility of tracking system based on the CDRA.

\subsubsection{Meet without having a call}

It is a fact that we may not meet all those we call and call all those we may meet. Thus an infected person may move in public places and all those who may come in contact with him/her may not obviously be traced. This is one of the main drawbacks of CDRA model.

\subsubsection{Call made through VOIP}

Technological advancement in VOIP, which provides calling facility is exponentially increasing day by day due to its economical rates. Some of the famous apps include, WhatsApp, Facebook Messenger, Snapchat, Viber, Skype, Telegram, WeChat, Line, etc. Since calls made on such apps are not registered in CDR log, therefore, it is not possible to track such cases using CDRA.

\subsection{Contact tracing framework}

Tracking model based on CDR only works once a suspect makes a call. In case where the COVID-19 suspect Meets another person without making a call and/or go to the crowded places then the model switches to tracking model based on contact tracing. Contact tracing model works on a low range wireless technology i.e., Bluetooth. The devices which come nearby exchanges random sequence. The log record of devices which are physically in contact are maintained in the cloud storage under the supervision of concern government department. Application programming interface (API) of the contact tracing is made under the umbrella of Google and Apple. One of the key attributes of this model is the privacy and security of the end users.

ENS based apps are continuously developed around the world in order to track and trace those people who come in contact with COVID-19 patients. A unique and random key of 4 digits is generated on a mobile device after a person chooses this model. The key is altered in a continuous manner after a time span of 10 to $20 \mathrm{~min}$. This feature 
helps to ensure that the location of an individual will remain unrecognized. If two phones with ENS based app come into contact with each other, then the random keys will be exchanged between the devices through BLE. As a consequence, the power consumption will be improved along with guaranteed privacy.

An individual phone checks the record of all random keys in a periodic manner to identify a COVID-19 patient. The user and the concerned department of government receive exposure notification on their phone in case of existence of any match. As a result, the limitations of CDR can be overcome if a person comes in physical contact with another person without any phone call, because privacy of an individual must be prioritized. It is important to mention that, a user opt out it any time after giving its own authorization in exposure notifications. The matching of exposure notification is only done in the device and the framework can share the identity with Google, Apple and different people. The concerned authorities can ask for contact details after taking the permission of a user. The overall execution of this model is explained in [37].

\subsubsection{Limitations of contact tracing}

Recently, numerous apps have been introduced for tracking COVID-19 patients and almost all apps are based on GPS tracking system or connectivity through BLE. These tracking apps require location services to be enabled to detect the devices in the range. Following are some of the major limitations and shortcomings of the apps, which are based on the contact tracing that are deployed for the tracking of the COVID-19 patients.

\subsubsection{Installation of apps}

The tracking app must be installed otherwise it will be difficult to track and trace any suspected patient.

\subsubsection{Privacy concerns}

As discussed earlier, almost all the tracking apps require location service to be "on" to detect devices within the range. This enforces everyone to turn on the location app by giving these apps and other apps access to one's location. Secondly, different private companies have made these apps and their source codes publicly inaccessible, therefore one cannot ensure the validity and security of these apps. Security and privacy of the data is of great concern, which are using these apps.

\subsubsection{Power consumption}

Power consumption by all these apps is another drawback. Mostly these apps drain out the battery quickly due to heavy power consumption. 


\subsubsection{Literacy}

In order to use such tracking apps, one has to be literate otherwise the user will not be able to use it. It is very rare to use such tracking apps by a large number of people. Understanding, installation and operation of such apps make them very rare to use. Since literacy rate of the under developed countries are very low, therefore, tracking system based on such apps is not considered to be a viable solution.

\section{Proposed model}

The use of VOIP calls has largely increased in recent years thereby replacing the cellular services. From most of the recently published research studies, the trend have moved beyond the overall usage of the Internet and now the focus is more on the use of social media [38-40].

Since calls made on VOIP are not registered in CDR log, therefore, it is not possible to track such cases using CDRA. In addition to this, if a call is made on such apps and mobile is not taken along, then the contact tracing strategy fails to track the COVID19 patients. In order to overcome the existing model limitations, the proposed model includes tracking of COVID-19 patients based on hybrid technologies. The proposed hybrid model includes cellular, cyber and low range wireless technologies as shown in Fig. 3.

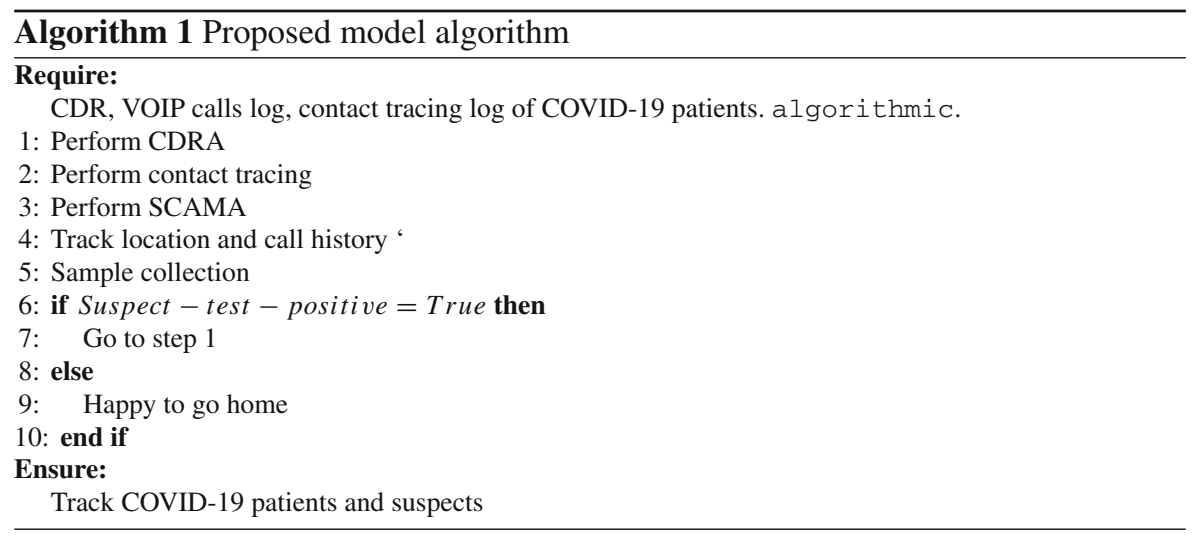

If the patient is diagnosed with COVID-19, the concerned government department will perform CDRA, contact tracing along with the VOIP calls analysis (VOIPCA) on the active SIM of the patient with his /her consent. After detail analysis of CDR, VOIP calls log and contact tracing log, the people with whom the COVID-19 patient met will be contacted by the concern government team. In order to check the status of these suspected patients, COVID-19 samples will be collected. Until COVID-19 test result is declared the suspects will be guided to remain stay at their home and properly keep themselves isolated. In case the test comes positive, the patient will be shifted to 


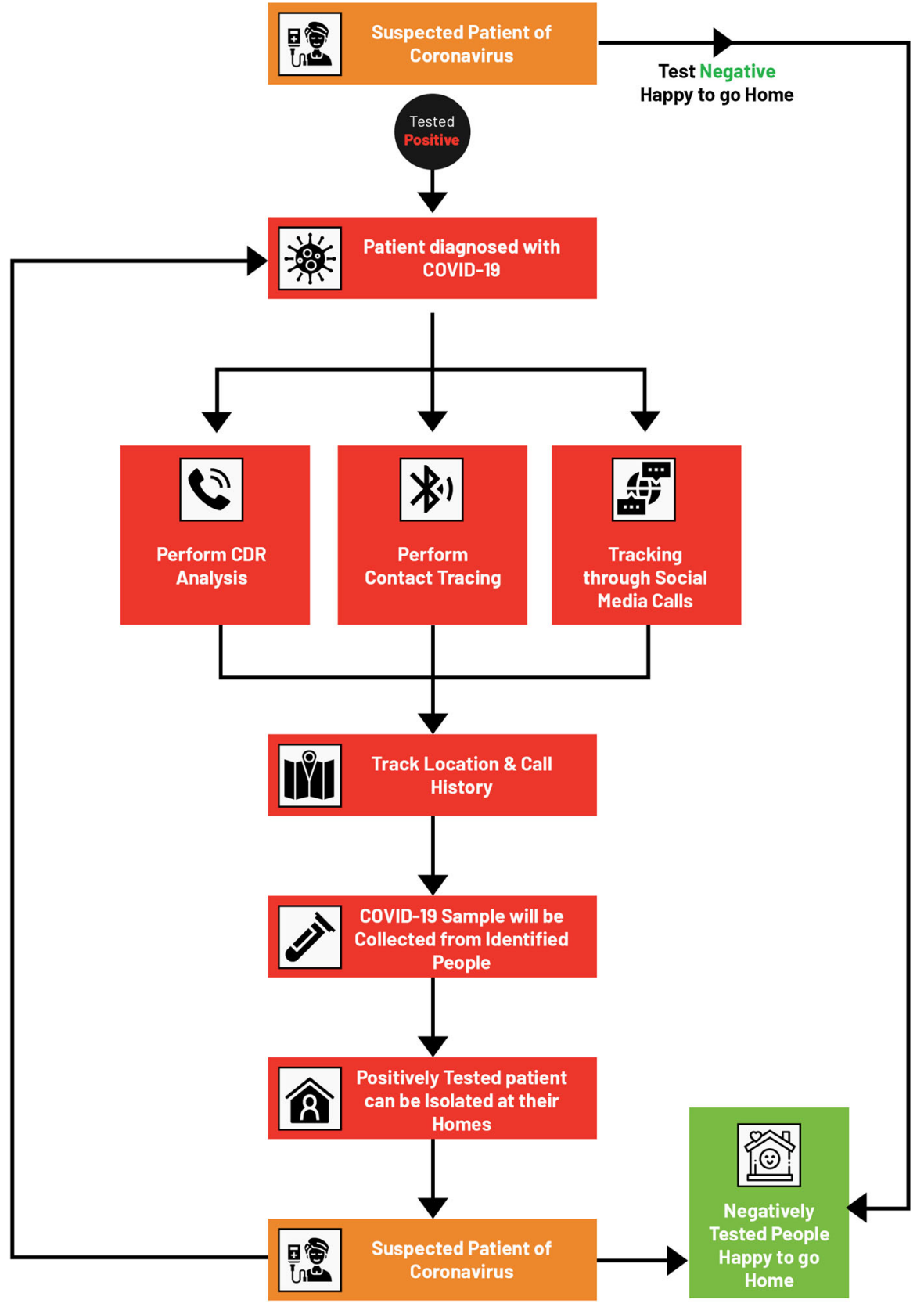

Fig. 3 Detail flowchart of proposed hybrid model 
Table 1 Relevant information contained in patient VOIP calls log

\begin{tabular}{lllll}
\hline Date and time & A party & B party & Call type & Call duration (mins) \\
\hline 08/01/2020 11:15:11 & 1234567890 & 12121212121 & Call outgoing & 14.03 \\
08/01/2020 13:12:11 & 1234567890 & 13131313131 & Call incoming & 14.23 \\
08/01/2020 15:30:24 & 1234567890 & 14141414141 & Call outgoing & 4.45 \\
09/01/2020 13:34:44 & 1234567890 & 15151515151 & Call outgoing & 1.03 \\
09/01/2020 20:13:13 & 1234567890 & 16161616161 & Call incoming & 2.11 \\
09/01/2020 22:12:12 & 1234567890 & 17171717171 & Call outgoing & 3.33 \\
10/01/2020 15:12:24 & 1234567890 & 18181818181 & Call outgoing & 4.22 \\
10/01/2020 16:23:24 & 1234567890 & 19191919191 & Call incoming & 3.32 \\
11/01/2020 13:14:22 & 1234567890 & 12121212121 & Call incoming & 5.34 \\
12/01/2020 23:24:55 & 1234567890 & 15151515151 & Call incoming & 13.23 \\
13/01/2020 17:18:34 & 1234567890 & 17171717171 & Call outgoing & 12.13 \\
13/01/2020 19:12:24 & 1234567890 & 14141414141 & Call incoming & 12.53 \\
15/01/2020 12:12:42 & 1234567890 & 12121212121 & Call outgoing & 4.33 \\
16/01/2020 13:12:15 & 1234567890 & 16161616161 & Call outgoing & 2.43 \\
20/01/2020 11:33:24 & 1234567890 & 19191919191 & Call outgoing & 1.32 \\
\hline
\end{tabular}

the hospital. The entire tracking process will be repeated for all the people with whom the patient has been interacted and reported positive.

\subsection{Case study}

The main targets of the proposed model can be realized by discussing a case study based on hypothesis. In this way, the desired results of the model can be assessed. When an individual is declared as COVID-19 patient, then the first and foremost step is to isolate the patient and perform the CDR of that individual. In addition to this, collect the data of VOIP calls log and contact tracing log of patient. Beside CDRA and contact tracing as discussed in [37], analysis on app calling log starts just after getting the log from the patient. After receiving the log, it is organized in the format given in Table 1. The table contained only those attributes which are helpful in tracking the COVID-19 patients.

This log of the subject contained all the relevant information through which one can easily track and trace the patient through his/her calling contacts. From log, we can get the information about the VOIP calls, which are made and received by the patient along with date and time. This log contains the duration of all calls made and received. Before starting the calling log analysis, irrelevant data is removed from the $\log$. Such irrelevant data can be easily removed by keeping the data of couple of weeks and delete all the past data log. This operation ensures to limit the log in the desired dates. By doing so, one can easily analyze few weeks data with high efficiency and accuracy. Tabular data log can be easily converted into graphical one by using different 
Fig. 4 Graphical interpretation of patient's VOIP $\log$

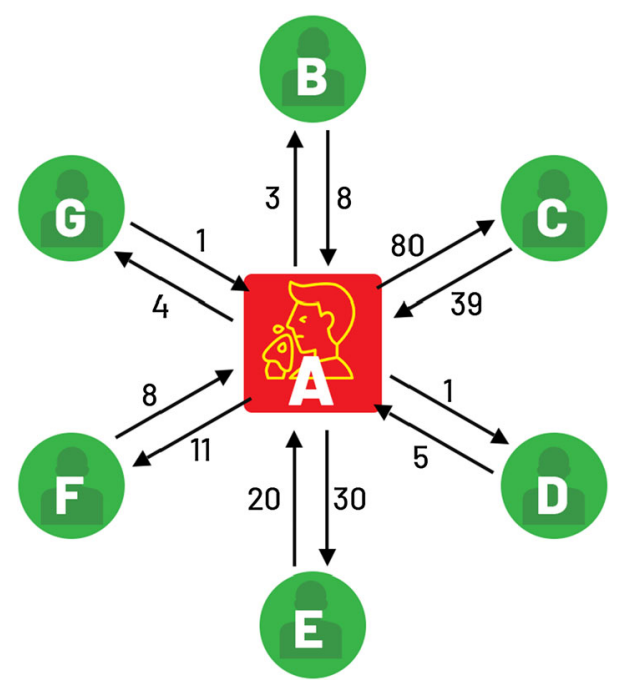

tools such as IBM I2 analyze as shown in Fig. 4. By doing so, one can easily extract the hidden patterns from the log. This will also improve the visualization of the data.

In Fig. 4, nodes represent the calling parties whereas the edges show the frequency of calls. Node A represents the subject, while nodes B, C, D, E, F and G are the one with whom the patient was in contact. From Fig. 4 one can easily interpret that the subject is frequently in contact with party $\mathrm{C}$, with a total of 80 outgoing calls and 39 incoming calls. The patient is in least contact with party G with only 4 outgoing calls and 1 incoming call. While investigating a COVID-19 patient, Fig. 4 depicts a vital role to memorize the patient with whom he/she has met. VOIP calls' log analysis outcomes will help the patient to reduce the chances to miss anyone with whom the patient has met. In fact all the parties those were in contact with the patient will be contacted. It will be confirmed whether they have met the patient or not. Through such analysis of a patient, one can easily improve the accuracy of tracking and tracing the suspect ones. After identification, the people who have met with the patient will be sent into the quarantine. Until the declaration of COVID-19 test result, the suspect is guided to be at home and remain isolated. In case of positive test the patient will be shifted to hospital. The entire tracking process will be repeated for all those people with whom the COVID-19 patient interacted.

After investigating patient $\mathrm{A}$ in the light of social app graphical links. During investigation let suppose patient $A$ admits the physical meeting with $C, D$ and $E$ in the infected period. Hence nodes E, C and D will be reported as suspects as shown in Fig. 5. The suspected nodes will be immediately connected by the concern government team to quarantine themselves unless the tests come negative.

After testing let node D comes positive. Node D will be labelled as a patient and the overall graph will be altered accordingly as shown in Fig. 6.

Now for node $\mathrm{D}$ the entire tracking process will be repeated i.e., app calling log analysis of node D as shown in Table 2. Its graphical interpretation is shown in Fig. 7. 
Fig. 5 Identification of COVID-19 suspects

Fig. 6 Identification of a COVID-19 patient
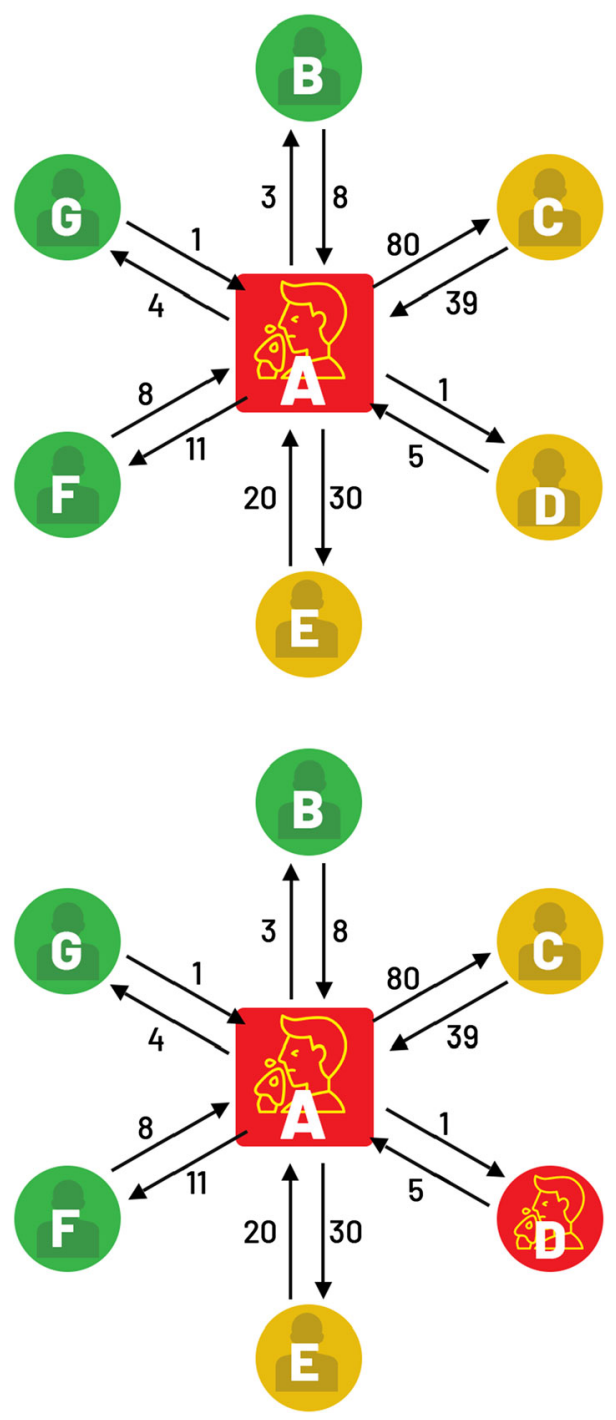

After investigating node D, one can easily find out the suspects in the light of app calling graph linkages as shown in Fig. 7. Similarly, the same cycle will be repeated to dig out the suspected ones as shown in Fig. 8. The proposed model will be keep on repeating until complete network of patients are tracked. By geo tagging the COVID-19 patients the concern government department can ensure the monitoring of the patients quarantine. In case of violation alerts will be sent to the concerned quarter.

The proposed model overcome all the limitations of the existing model by adding the additional feature of tracing through VOIP calls log as shown in Table 3. The capabilities of the proposed model is summarized in Table 4. The proposed tracking model only fails to track if no call is made neither through cellular network nor through 
Table 2 Relevant information contained in patient VOIP calls log (node D)

\begin{tabular}{llllc}
\hline Date and time & A party & B party & Call type & Call duration (mins) \\
\hline 05/01/2020 11:13:04 & 9876543210 & 21121212121 & Call outgoing & 3.34 \\
06/01/2020 13:15:04 & 9876543210 & 41141414141 & Call outgoing & 23.22 \\
06/01/2020 15:16:44 & 9876543210 & 51151515151 & Call outgoing & 3.12 \\
08/01/2020 10:23:14 & 9876543210 & 61161616161 & Call outgoing & 4.35 \\
08/01/2020 15:44:34 & 9876543210 & 81181818181 & Call outgoing & 1.22 \\
09/01/2020 14:34:43 & 9876543210 & 91191919191 & Call outgoing & 9.64 \\
10/01/2020 19:22:33 & 9876543210 & 31131313131 & Call outgoing & 5.88 \\
12/01/2020 18:45:24 & 9876543210 & 11111112121 & Call outgoing & 7.11 \\
13/01/2020 15:24:21 & 9876543210 & 51151515151 & Call outgoing & 3.65 \\
15/01/2020 14:15:25 & 9876543210 & 81181818181 & Call outgoing & 1.11 \\
15/01/2020 15:15:44 & 9876543210 & 31131313131 & Call outgoing & 4.90 \\
17/01/2020 16:34:42 & 9876543210 & 21121212121 & Call outgoing & 5.55 \\
19/01/2020 13:34:31 & 9876543210 & 61161616161 & Call outgoing & 6.44 \\
22/01/2020 20:13:32 & 9876543210 & 31131313131 & Call outgoing & 4.37 \\
\hline
\end{tabular}

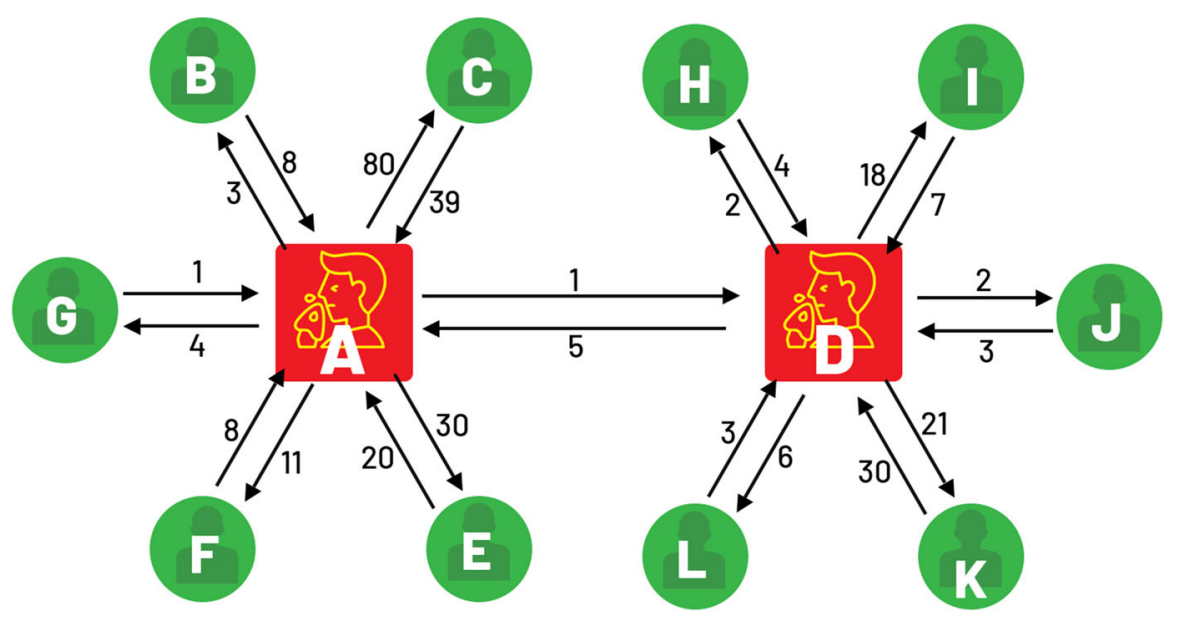

Fig. 7 Graphical interpretation of node D app calling log

VOIP services, and the contact tracing is also disabled. In such rare case the proposed model is not capable to track the COVID-19 patients.

\section{Conclusion}

Until the availability of any drug and vaccine for COVID-19 and similar kind of pandemics, the best solution will be to reduce the spread and impact of pandemic. This paper presents a novel model to overcome the spread of COVID-19 using contact 


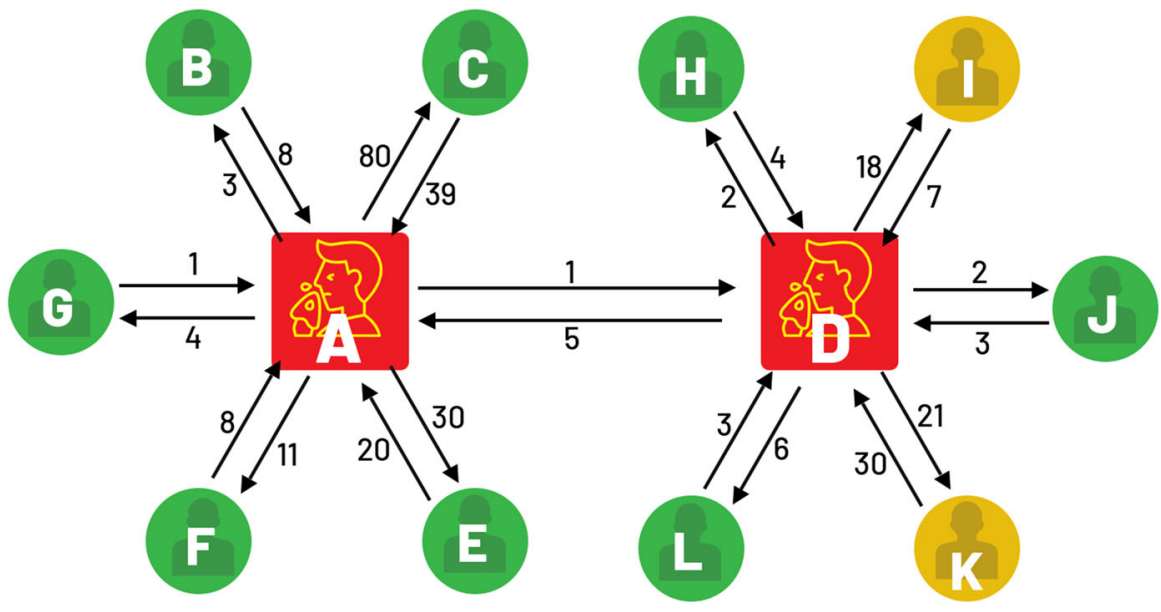

Fig. 8 Identification of COVID-19 suspects

Table 3 Comparison of proposed model

\begin{tabular}{llll}
\hline Tracking capabilities & {$[32]$} & {$[37]$} & Our work \\
\hline Tracing through call history & $x$ & $\checkmark$ & $\checkmark$ \\
Tracing without call history & $\checkmark$ & $\checkmark$ & $\checkmark$ \\
Tracing through VOIP calls & $x$ & $x$ & $\checkmark$ \\
Tracing through call history (suspect) & $x$ & $\checkmark$ & $\checkmark$ \\
Tracing without call history (suspect) & $\checkmark$ & $\checkmark$ & $\checkmark$ \\
Tracing through social media calls (suspect) & $x$ & $x$ & $\checkmark$ \\
Patient monitoring & $x$ & $\checkmark$ & $\checkmark$ \\
Path traversed by COVID-19 patients & $x$ & $\checkmark$ & $\checkmark$ \\
\hline
\end{tabular}

Table 4 Capability of proposed model

\begin{tabular}{llc}
\hline Tracking capabilities & Technology used & Status \\
\hline Trace and Track of COVID-19 patients having call history & CDRA & $\checkmark$ \\
Trace and Track of COVID-19 patients without call history & Contact tracing & $\checkmark$ \\
Trace and Track of COVID-19 patients having VOIP calls log & Tracing through social calls & $\checkmark$ \\
Trace and Track of COVID-19 suspects having call history & CDRA & $\checkmark$ \\
Trace and Track of COVID-19 suspects without call history & Contact tracing & $\checkmark$ \\
Track and Trace of COVID-19 suspects having VOIP calls log & Tracing through social calls & $\checkmark$ \\
Monitoring of COVID-19 patients & Tagging & $\checkmark$ \\
Path alerts traversed by COVID-19 patients & CDRA & $\checkmark$ \\
\hline
\end{tabular}


tracing, CDRA and VOIPCA. The proposed model can trace and track the infected COVID-19 patients effectively. The information of a patient and his/her interaction with suspected people can easily be retrieved by following this model. In this way, the model can successfully identify the individuals infected by COVID-19 along with possible suspects. In addition to this, the path followed by patients during the last few weeks can be traced and alerts can be sent to the public accordingly in order to avoid that particular path. It has been discussed in detail to demonstrate the effectiveness and implementation of proposed model. A contact tracing strategy is also utilized to track the path used by the COVID-19 patient without any call history. If the call is made on VOIP then the proposed model traces the patient through VOIPCA. Spreading of COVID-19 disease can be easily control by extracting information using hybrid model. The potential outcomes of the proposed model are tracking of COVID-19 patients, monitoring of isolated patients, tracking of suspected ones and inform the mass about the safest path to use. The proposed model can be easily used by the concern government team to minimize or stop the spread of COVID-19 disease. In future, the authors are planning to automate the entire process of tracking and tracing system. An app will be built to automatically perform all the simulations for tracking and tracking of COVID-19 patients.

\section{References}

1. Yee J, Unger L, Zadravecz F, Cariello P, Seibert A, Johnson MA, Fuller MJ (2020) Novel coronavirus 2019 (COVID-19): emergence and implications for emergency care. J Am Coll Emerg Phys Open 1:63-69

2. Park M, Cook AR, Lim JT, Sun Y, Dickens BL (2020) A systematic review of COVID-19 epidemiology based on current evidence. J Clin Med 9(4):967

3. Bradbury-Jones C, Isham L (2020) The pandemic paradox: the consequences of COVID-19 on domestic violence. J Clin Nurs 29:2047-249

4. Di Gennaro F, Pizzol D, Marotta C, Antunes M, Racalbuto V, Veronese N, Smith L (2020) Coronavirus diseases (COVID-19) current status and future perspectives: a narrative review. Int J Environ Res Public Health 17(8):2690

5. Norman G (2020) The once and future myths of medical education. J Grad Med Educ 12(2):125-130

6. Hassan SA, Sheikh FN, Jamal S, Ezeh JK, Akhtar A (2020) Coronavirus (COVID-19): a review of clinical features, diagnosis, and treatment. Cureus 12(3):e7355

7. Cavallo JJ, Forman HP (2020) The economic impact of the COVID-19 pandemic on radiology practices. Radiology 296:201495

8. Rana S (2020) An unprecedented footprint of covid19 on education sector: a review on impact and measures. UGC CARE J 31(23):548-551

9. Hoque A, Shikha FA, Hasanat MW, Arif I, Hamid ABA (2020) The effect of coronavirus (COVID-19) in the tourism industry in china. Asian J Multidiscip Stud 3(1):52-58

10. Nicola M, Alsafi Z, Sohrabi C, Kerwan A, Al-Jabir A, Iosifidis C, Agha M, Agha R (2020) The socioeconomic implications of the coronavirus and COVID-19 pandemic: a review. Int J Surg 78:185-193

11. Albulescu C (2020) Coronavirus and oil price crash: a note. arXiv preprint arXiv:2003.06184

12. Sahoo PP, Rath S (2020) Potential impact of corona virus on agriculture sector. Biot Res Today 2(4):6465

13. Poudel PB, Poudel MR, Gautam A, Phuyal S, Tiwari CK, Bashyal N, Bashyal S (2020) COVID-19 and its global impact on food and agriculture. J Biol Today's World 9:221

14. Ansari B, Ganjoo M (2020) Impact of COVID-19 on advertising: a perception study on the effects on print and broadcast media and consumer behavior. UGC CARE J 31(28):52-62

15. Schiffbauer MT (2020) Western Balkans regular economic report, no. 17, spring 2020: the economic and social impact of COVID-19. World Bank, Washington, DC 
16. Fetzer T, Hensel L, Hermle J, Roth C (2020) Coronavirus perceptions and economic anxiety. arXiv preprint arXiv:2003.03848

17. Sohrabi C, Alsafi Z, O’Neill N, Khan M, Kerwan A, Al-Jabir A, Iosifidis C, Agha R (2020) World health organization declares global emergency: a review of the 2019 novel coronavirus (COVID-19). Int J Surg 76:71-76

18. Parnell D, Widdop P, Bond A, Wilson R (2020) COVID-19, networks and sport. Managing Sport and Leisure 1-7, Advance online publication. https://doi.org/10.1080/23750472.2020.1750100

19. Armitage R, Nellums LB (2020) COVID-19 and the consequences of isolating the elderly. Lancet Public Health 5(5):e256

20. Cascella M, Rajnik M, Cuomo A, Dulebohn SC, Di Napoli R (2020) Features, evaluation and treatment coronavirus (COVID-19). Stat Pearls https://www.ncbi.nlm.nih.gov/books/NBK554776/

21. World Health Organization et al (2019) Coronavirus disease (COVID-19) technical guidance: Laboratory testing for 2019-nCov in humans. [Cited 2020 Apr 12]. Disponible en https://www.who.int/ emergencies/diseases/novel-coronavirus-2019/technical-guidance/laboratory-guidance

22. Ahmed N, Michelin RA, Xue W, Ruj S, Malaney R, Kanhere SS, Seneviratne A, Hu W, Janicke H, Jha SK (2020) A survey of COVID-19 contact tracing apps. IEEE Access 8:134577-134601

23. Ekong I, Chukwu E, Chukwu M (2020) COVID-19 mobile positioning data contact tracing and patient privacy regulations: exploratory search of global response strategies and the use of digital tools in Nigeria. JMIR mHealth uHealth 8(4):e19139

24. Buchanan WJ, Imran MA, Ur-Rehman M, Zhang L, Abbasi QH, Chrysoulas C, Haynes D, Pitropakis N, Papadopoulos P (2020) Review and critical analysis of privacy-preserving infection tracking and contact tracing. arXiv preprint arXiv:2009.05126

25. Horak R (2007) Telecommunications and data communications handbook. Wiley, New York

26. Petersen JK (2002) The telecommunications illustrated dictionary. CRC Press, Boca Raton

27. Khan S, Ansari F, Dhalvelkar HA, Computer S (2017) Criminal investigation using call data records (CDR) through big data technology. In: 2017 International conference on nascent technologies in engineering (ICNTE). IEEE, pp 1-5

28. Kedma G, Guri M, Sela T, Elovici Y (2013) Analyzing users' web surfing patterns to trace terrorists and criminals. In: 2013 IEEE International conference on intelligence and security informatics. IEEE, pp 143-145

29. He Y, Yu FR, Zhao N, Yin H, Yao H, Qiu RC (2016) Big data analytics in mobile cellular networks. IEEE Access 4:1985-1996

30. Kumar M, Hanumanthappa M, Suresh Kumar TV (2017) Crime investigation and criminal network analysis using archive call detail records. In: 2016 Eighth international conference on advanced computing (ICoAC). IEEE, pp 46-50

31. Abba E, Aibinu AM, Alhassan JK (2019) Development of multiple mobile networks call detailed records and its forensic analysis. Digit Commun Netw 5(4):256-265

32. Google (2020) Exposure notification API launches to support public health agencies https://www. googlenewsapp.com/exposure-notification-apilaunches-to-support-public-health-agencies/

33. Apple (2020) Exposure notification-apple developer documentation https://developer.apple.com/ documentation/exposurenotification

34. Google (2020) Exposure notifications: helping fight COVID-19-google https://www.google.com/ covid19/exposurenotifications/

35. Apple (2020) Privacy-preserving contact tracing. https://covid19.apple.com/contacttracing

36. Apple and Google (2020) Contact tracing-bluetooth specification. https://coregroup.org/covidresource-lib/apple-and-googlescontact-tracing-bluetooth-spec-for-covid-19-explained/

37. Nisar S, Zuhaib MA, Ulasyar A, Tariq M (2020) A privacy preserved and cost efficient control scheme for coronavirus outbreak using call data record and contact tracing. IEEE Consum Electron Mag 10:1

38. Pulido CM, Redondo-Sama G, Sordé-Martí T, Flecha R (2018) Social impact in social media: a new method to evaluate the social impact of research. PLoS ONE 13(8):e0203117

39. Kapoor KK, Tamilmani K, Rana NP, Patil P, Dwivedi YK, Nerur S (2018) Advances in social media research: past, present and future. Inf Syst Front 20(3):531-558

40. Akakandelwa A, Walubita G (2018) Students' social media use and its perceived impact on their social life: a case study of the University of Zambia 1-14. http://palevel.unza.zm/handle/123456789/5247

Publisher's Note Springer Nature remains neutral with regard to jurisdictional claims in published maps and institutional affiliations. 